DRAFT VERSION JULY 18, 2007

Preprint typeset using $\mathrm{LT}_{\mathrm{E}} \mathrm{X}$ style emulateapj v. 6/22/04

\title{
INTERMEDIATE-MASS BLACK HOLES IN COLLIDING CLUSTERS: IMPLICATIONS FOR LOWER-FREQUENCY GRAVITATIONAL-WAVE ASTRONOMY
}

\author{
Pau Amaro-SeoAne ${ }^{1}$, Marc Freitag ${ }^{2}$ \\ Draft version July 18, 2007
}

\begin{abstract}
Observations suggest that star clusters often form in binaries or larger bound groups. Therefore, mergers between two clusters are likely to occur. If these clusters both harbor an intermediate-mass black hole (IMBH; $\sim 10^{2-4} M_{\odot}$ ) in their center, they can become a strong source of gravitational waves when the black holes merge with each other. In order to understand the dynamical processes that operate in such a scenario, one has to study the evolution of the merger of two such young massive star clusters, and more specifically, their respective IMBHs. We employ the direct-summation NBODY4 numerical tool on special-purpose GRAPE6 hardware to simulate a merger of two stellar clusters each containing 63,000 particles and a central IMBH. This allows us to study accurately the orbital evolution of the colliding clusters and the embedded massive black holes. Within $\sim 7 \mathrm{Myr}$ the clusters have merged and the IMBHs constitute a hard binary. The final coalescence happens in $\sim 10^{8} \mathrm{yrs}$. The implication of our analysis is that intermediate-mass black holes merging as the result of coalescence of young dense clusters could provide a source for the Laser Interferometer Space Antenna (LISA) space-based gravitational wave detector mission. We find that interactions with stars increase the eccentricity of the IMBH binary to about 0.8 . Although the binary later circularizes by emission of gravitational waves, the residual eccentricity can be detectable through its influence on the phase of the waves if the last few years of inspiral are observed. For proposed higher-frequency space-based missions such as the Big Bang Observer $(B B O)$, whose first purpose is to search for an inflation-generated gravitational waves background in the $10^{-1}-1 \mathrm{~Hz}$ range, binary IMBH inspirals would be a foreground noise source. However, we find that the inspiral signals could be characterized accurately enough that they could be removed from the data stream and in the process provide us with detailed information about these astrophysical events.
\end{abstract}

Subject headings: Black hole physics, gravitational waves, stellar dynamics, methods: N-body simulations

\section{INTRODUCTION}

There are at least two lines of evidence indicating that stellar clusters, containing $10^{4}-10^{6}$ stars, can merge with each other relatively early in there evolution. First, high-resolution Hubble Space Telescope observations of the Antennæ (Whitmore et al. 1999; Zhang \& Fall 1999) or Stephan's Quintet Gallagher et al. 2001) reveal hundreds of young massive star clusters in the star forming regions. These clusters are clustered into larger complexes of a few $100 \mathrm{pc}$. Since they harbor $\sim 10^{5}$ stars within a few parsecs and are older than $5 \mathrm{Myr}$, they are most likely bound clusters. These cluster complexes have been suggested as the progenitors of ultra-compact dwarf galaxies (UCDGs), as a result of the amalgamation of tens or hundreds of their member clusters (Fellhauer \& Kroupa 2002, 2005). Furthermore, in more quiescent environments, a significant fraction of clusters may form as bound binaries or low-order multiples. Observationally, this is indicated by the large number of young binary clusters observed in the Magellanic Clouds (MCs). For instance, Dieball et al. (2002) estimate that about one cluster in eight in the Large Magellanic Cloud is a member of a bound group. Most clusters in (candidate) binaries are coeval and younger than $300 \mathrm{Myr}$, suggesting that binary clusters generally merge early. Another indication that mergers may be common, at least in environments such as the MCs, is that the clusters in these galaxies are significantly flattened (e.g. Kontizas et al. 1989. 1990: van den Bergh 1991), possibly as a result of rotation that could stem from a merger. The typical lack of significant rotation in Galactic or M31 globular clusters (Han \& Ryden 1994) does

\footnotetext{
1 (PAS) Max Planck Intitut für Gravitationsphysik (Albert-EinsteinInstitut), D-14476 Potsdam, Germany

2 (MF) Institute of Astronomy, University of Cambridge, Madingley Road, CB3 0HA Cambridge, UK
}

not invalidate the idea that a large fraction of them may also be merger products; the more intense tidal field of the Galaxy may lead to faster decrease of the rotation. Theoretically, binary clusters are predicted to be a common outcome of the off-center collision of two molecular clouds (Fujimoto \& Kumai 1997; Bekki et al. 2004) or the collapse of a spherical shell of stars whose formation is triggered by a SN explosion in a molecular cloud (Theis 2002).

In this paper, we consider the possibility that the merging clusters each contain an intermediate-mass black hole (IMBH; mass $M \sim 10^{2-4} M_{\odot}$ ) and investigate the consequences for gravitational-wave (GW) astronomy. The observational evidence for IMBHs in clusters is only suggestive van der Marel 2004). However several authors have shown how mass segregation leads in sufficiently compact young clusters to a phase of runaway collisions among the heaviest stars, possibly leading to the formation of an IMBH (Portegies Zwart \& McMillan 2000: Gürkan et al.|2004: Portegies Zwart et al. 2004; Freitag et al. 2006b).

\section{FORMATION OF A MERGED CLUSTER}

To form a realistic merged stellar cluster we make two clusters collide on a parabolic orbit so that the minimum distance at which they pass by is $d_{\min }$ if they are considered to be point particles at their respective centers of mass. We chose for our fiducial simulation $d_{\min }=2 \mathrm{pc}$, corresponding to a relative velocity at pericenter of $23.3 \mathrm{~km} \mathrm{~s}^{-1}$. Given that the clusters are set initially on a parabolic orbit, the inevitable loss of energy (to, e.g., escaping stars) means that they will always merge to form a larger cluster. Each cluster contains $\mathcal{N}_{\star}=6.3 \times 10^{4}$ particles of $1 \mathrm{M}_{\odot}$ each which are distributed according to King models with $W_{0}=7$ (cluster 1) and $W_{0}=6$ (cluster 2). Their core radii are $R_{\text {core } 1}=0.203 \mathrm{pc}$ and $R_{\text {core } 2}=0.293 \mathrm{pc}$. Thus, they are compact enough to have experienced an early 


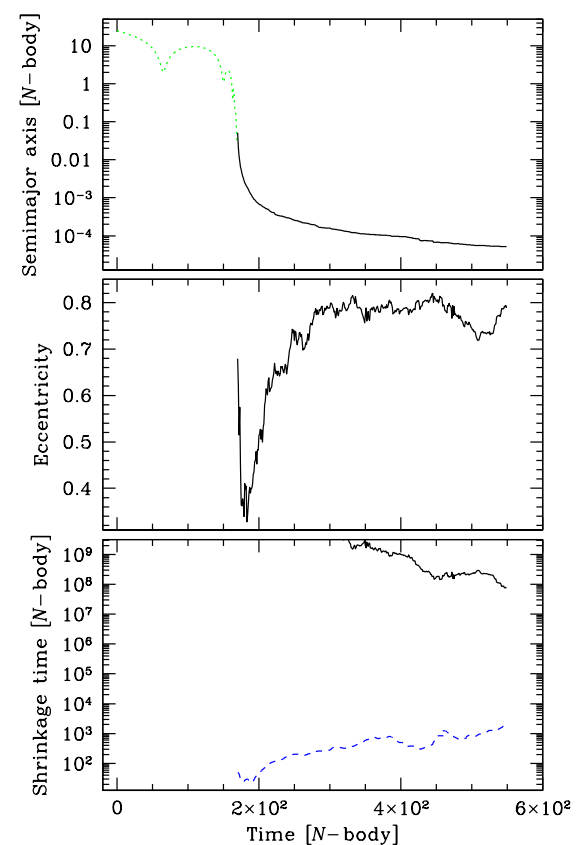

FIG. 1. - Evolution of the IMBH binary of the $N$-body simulation. In the upper panel, we show the shrinkage of the semi-major axis as a solid line. The initial part of the curve in dashes represents the separation between the IMBHs before they have formed a bound binary. In the middle panel we display the evolution of the eccentricity, which increases to $\sim 0.8$. The bottom panel gives a an indication of the relevance of GW emission in the evolution of the binary in comparison with stellar dynamical processes: the solid line corresponds to the timescale for GWs emission (Peters 1964) and the dashed curve indicates the dynamical shrinkage timescale, given by $a / \dot{a}$, where $a$ is the time derivative of $a$. One sees that at the end of the simulation when $a \simeq 5 \times 10^{-5} \mathrm{pc}, \mathrm{GW}$ emission is still less efficient than interaction with stars by several orders of magnitude.

core collapse that may lead to IMBH formation according to Gürkan et al. (2004) and Freitag et al. (2006b) who find that for clusters with a moderate initial concentration $\left(W_{0} \sim 6-7\right)$, a half-mass radius smaller than $1-2 \mathrm{pc}$ is required. We note that our results, to be presented ahead, can be rescaled to more extended clusters. The central velocity dispersions are of $\sigma_{\text {core } 1}=8.41 \mathrm{~km} \mathrm{~s}^{-1}$ and $\sigma_{\text {core } 2}=8.29 \mathrm{~km} \mathrm{~s}^{-1}$. Both clusters harbor an intermediate-mass black hole (IMBH) with a mass of $300 \mathrm{M}_{\odot}$, so that the total particle number is $\mathcal{N}_{\star}=126002$. The calculations were performed on special-purpose GRAPE6A PCI cards with the direct-summation NBODY 4 code of Aarseth (2003). The particular advantage of this code for our study is Aarseth's close-encounter regularization scheme, which is free of any softening. The peak performance of these cards is of 130 Gflop, roughly equivalent to 100 single PCs, which makes possible simulations of stellar clusters with a realistic particle number.

\section{EVOLUTION OF THE IMBH BINARY}

In order to have a global view of the evolution of the system, in Fig. (1) we present the dynamical evolution of the IMBH binary (hereafter "binary"). The length and time units used are $R_{\mathrm{NB}}=1 \mathrm{pc}$ and $t_{\mathrm{NB}}=4.16 \times 10^{4} \mathrm{yr}$. In Fig. (2) we show both the semi-major axis of the binary and its orbital period versus its eccentricity. Whilst the direct summation simulations provide us with a highly accurate description of the orbital parameters of the binary as it approaches the LISA band, the required computational effort to follow the evolution up to the very final stage of coalescence is unjustified. Therefore in Fig. 2 we

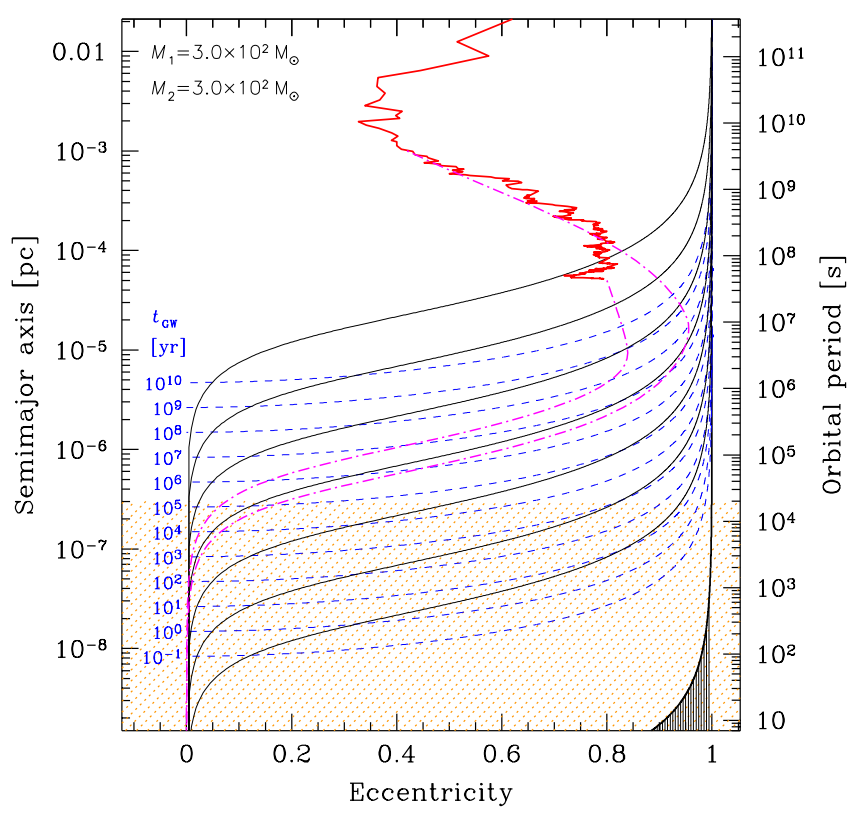

FIG. 2.- Inspiral of an IMBH binary followed in the eccentricity-semimajor axis plane. The jagged solid line (in red in the color online version) is the result of the $N$-body simulation. The dash-dotted lines (in magenta) are results of the analytical model (equations 13 for two sets of parameters. In one case, we use $K_{0}=0.5$ and start at an early stage, $a=10^{-3} \mathrm{pc}, e=0.42$. The other track is for $K_{0}=0.1$ and starts with the final values of the $N$-body run, $a=5 \times 10^{-5} \mathrm{pc}, e=0.8$. In both cases the parameters for the stellar background -estimated from the $N$-body run- are set to $\sigma=23 \mathrm{~km} \mathrm{~s}^{-1}$ and $\rho=1.6 \times 10^{5} \mathrm{M}_{\odot} \mathrm{pc}^{-3}$. The smooth solid (black) lines are trajectories for evolution by GW emission (Peters 1964) and the dashed (blue) lines indicate the corresponding timescale, $t_{\mathrm{GW}}$ (labels on the left). The dark dashed area, at the bottom right, indicates approximately the region of unstable orbits. Finally the light (orange) shaded area shows when the $n=2$ harmonic of the GW signal is in the LISA bandwidth $\left(P_{\text {orb }}<2 \times 10^{4} \mathrm{~s}\right)$.

stop the direct-summation calculation after the initial strongly fluctuating phase; when the eccentricity achieves a large and seemingly steady value of $\sim 0.8$. We note that an eccentricity larger than 0.65 is reached in additional simulations we have performed with slightly different initial conditions and that a similar result $(e \simeq 0.8)$ was found in a simulation of a merger of two clusters of 10,000 particles each using a special regularization method for the massive binary (S. Aarseth, personal communication; see Mikkola \& Aarseth 2002 for the numerical method). In order to determine the binary properties in the LISA frequency band, we employ the results of the $N$-body simulation at $t \simeq 550 t_{\mathrm{NB}} \simeq 23 \mathrm{Myr}$ and extend it with a analytical method down to the moment in which the binary enters LISA band. The basic idea is to split the evolution of the semi-major axis and the eccentricity into two contributions, one driven by the dynamical interactions with stars (subscript dyn) and the other due to emission of GWs (subscript GW),

$$
\frac{d a}{d t}=\left.\frac{d a}{d t}\right|_{\mathrm{dyn}}+\left.\frac{d a}{d t}\right|_{\mathrm{GW}}, \frac{d e}{d t}=\left.\frac{d e}{d t}\right|_{\mathrm{dyn}}+\left.\frac{d e}{d t}\right|_{\mathrm{GW}}
$$

The "GW" terms are as given in Peters (1964). Using the relationships of Ouinlan (1996), we have that since $d(1 / a) /\left.d t\right|_{\mathrm{dyn}}=-a^{-2} d a / d t=-H G \rho / \sigma$,

$$
\left.\frac{d a}{d t}\right|_{\mathrm{dyn}}=-H \frac{G \rho}{\sigma} a^{2} .
$$




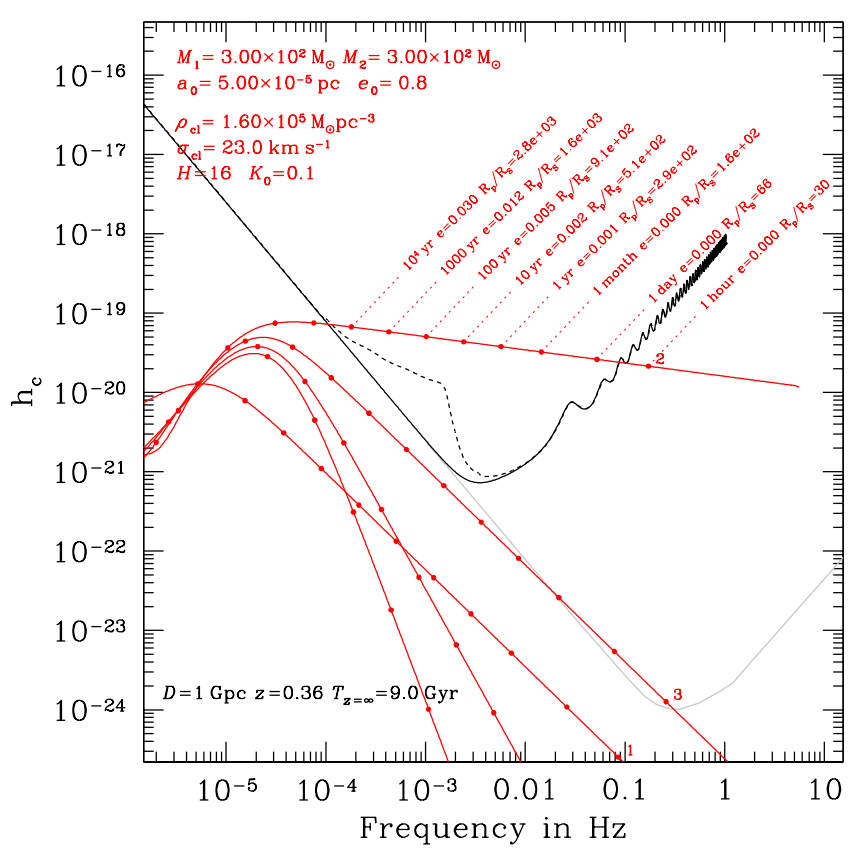

FIG. 3.- Characteristic amplitude of the first five harmonics of the quadrupolar gravitational radiation emitted during the inspiral of an $300 \mathrm{M}_{\odot}+300 \mathrm{M}_{\odot}$ binary. The orbit is evolved according to Eq. 1 starting with the conditions similar to those reached at the end of the fiducial simulation, i.e., $e=0.8, a=5 \times 10^{-5} \mathrm{pc}$ with an ambient stellar density $\rho_{\mathrm{cl}}$ and (1-D) velocity dispersion $\sigma_{\mathrm{cl}}$. We assume the source is at a distance $D=1 \mathrm{Gpc}$. We indicate the noise curve $\sqrt{f S_{h}(f)}$ for LISA (Larson et al. 2000; Larson 2003), with the Galactic binary white dwarf confusion background in dashed line (Bender \& Hils 1997), and (in grav) for the BBO sensitivity curve (Cutler \& Harms 2006). We label the position of the source at various times before plunge. Note that the height of the point for the amplitude above the $L I S A / B B O$ curve does not represent the SNR (see text).

In this last expression, $G$ is the gravitational constant, $\rho$ is the density, $\sigma$ is the velocity dispersion and $H$ is the hardening constant (Ouinlan 1996). We measure $H \simeq 16$, in agreement with previous works (Ouinlan 1996; Sesana et al. 2004). For a hard enough binary, $d e /\left.d \ln (1 / a)\right|_{\text {dyn }}=K(e)$ and so

$$
\left.\frac{d e}{d t}\right|_{\mathrm{dyn}}=H \frac{G \rho}{\sigma} a K(e)
$$

with $K(e) \sim K_{0} e\left(1-e^{2}\right)$ (Merritt \& Milosavliević 2005 and references therein). We choose the value of $K_{0}=0.1$ for matching purposes with the direct-summation $N$-body simulation. During the run, the ratio $\rho / \sigma$ decreases by less than $30 \%$ at the radius of influence of the binary so we assume it is constant. The evolution is dominated by $\mathrm{GW}$ emission during the last $10^{8}$ yrs (the evolution from the moment the binary is bound takes $\sim 160 \mathrm{Myr}$ ) and enters the LISA bandwidth with $e \simeq 0.07$. If we employ the fiducial value of $K_{0}=0.5$ (Merritt $\&$ Milosavljević 2005) but start earlier on the same curve (at a lower eccentricity), we find a evolution which fits the initial part of the $N$-body run satisfactorily but produces too large an eccentricity at later times.

\section{DETECTION BY LISA AND THE BBO}

In the late phase of its inspiral, a binary may become a detectable source of GWs. The characteristic amplitude of the gravitational radiation from a source emitting at frequency $f$ is $h_{\mathrm{c}}=(\pi D)^{-1}(2 \dot{E} / \dot{f})^{1 / 2}$, where $D$ is the distance to the source, $\dot{E}$ is the power emitted and $\dot{f}$ the time derivative of the frequency (Finn \& Thorne 2000). With this definition, the signal-to-noise ratio (SNR) of an event is obtained, assuming ideal signal processing, by the integral $(\mathrm{SNR})^{2}=$ $\int_{f_{1}}^{f_{2}}\left[h_{\mathrm{c}}^{2}(f) / f S_{h}(f)\right] d(\ln f)$, where $f_{1}$ and $f_{2}$ are the initial and final frequencies of the source during the observation and $S_{h}(f)$ is the instrumental noise of the detector at frequency $f$ (Phinnev 2002; Barack \& Cutler 2004).

In Fig. 3 we follow the signal emitted by the IMBHs of the fiducial simulation during their GW-driven inspiral. We plot the five lowest harmonics of the quadrupolar emission (Peters $\&$ Mathews 1963). In this figure, we assume a distance of $1 \mathrm{Gpc}$. Both for LISA and the $B B O$, only the $n=2$ harmonic is detectable, during the last few years of inspiral. However, the small residual excentricity induces a difference in the phase evolution of the $n=2$ signal compared to a circular inspiral. If the source is followed from a time $t_{\mathrm{mrg}}$ before merger until merger, the accumlated phase shift is

$$
\Delta \psi_{e} \simeq 1.0\left(\frac{e_{10^{-4} \mathrm{~Hz}}}{0.05}\right)^{2}\left(\frac{t_{\mathrm{mrg}}}{1 \mathrm{yr}}\right)^{17 / 12}\left(\frac{\mathcal{M}_{z}}{1000 \mathrm{M}_{\odot}}\right)^{25 / 36},
$$

where $e_{10^{-4} \mathrm{~Hz}}$ is the eccentricity when the $n=2$ signal has reached a frequency of $10^{-4} \mathrm{~Hz}$ and $\mathcal{M}_{z} \equiv(1+$ $z)\left(M_{1} M_{2}\right)^{3 / 5}\left(M_{1}+M_{2}\right)^{-1 / 5}$ is the redshifted chirp mass (Cutler \& Harms 2006). For our fiducial case (with $e_{10^{-4} \mathrm{~Hz}}=0.07$ ), the eccentricity should be detectable $\left(\Delta \psi_{e} \geq 2 \pi\right)$ if observations span at least the last 3-4 years before merger. A measured non-zero eccentricity is an important constraint on the formation process and stellar environment of the binary. The $B B O$ could detect a $300 \mathrm{M}_{\odot}+300 \mathrm{M}_{\odot}$ inspiral to at least a redshift of 17 but the residual eccentricity will not be measurable $\left(t_{\mathrm{mrg}} \leq 1\right.$ day) and significantly more massive IMBHs cannot be seen because their GWs are redshifted to too low frequencies.

We now proceed to estimate roughly the detection rate of binary IMBHs. Fregeau et al. (2006) have recently considered the detection of binary IMBHs by LISA, assuming that, generically, two IMBHs form in any cluster undergoing a collisional runaway, as suggested by the stellar dynamical simulations of Gürkan et al. (2006). Here we assume that the runaway process leads to the formation of only one IMBH; indeed it is not clear whether the two collision-grown very massive stars (VMSs, with $m_{*} \gg 100 \mathrm{M}_{\odot}$ ) can avoid merging with each other before they become IMBHs. Compared to the scenario considered by these authors, ours involve one more step, namely, the merger of a IMBH-hosting cluster with another one. Schematically, the computation of the detection rate for both scenarios can be written

$$
\Gamma=\frac{4 \pi}{3} D_{\text {max }}^{3} \times \dot{n}_{\text {clust }} \times P_{\text {bin }} .
$$

$D_{\max }$ is the distance at which a coalescence event can be detected, $\dot{n}_{\text {clust }}$ is the rate of formation per unit volume of clusters massive enough for (potential) IMBH formation. Rough estimates for these terms are $D_{\max } \approx 1-3 \mathrm{Gpc}$ and $\dot{n}_{\text {clust }} \gtrsim$ $5 \times 10^{-10} \mathrm{Mpc}^{-3} \mathrm{yr}^{-1}$ (Fregeau et al. 2006). $P_{\text {bin }}$ is the probability that a cluster will host a (coalescing) IMBH binary. Only this term differs between the two scenarios. In our case, it can be written as

$$
P_{\text {bin }}=P_{\text {merg }} P_{\text {ra }}^{2} P_{\text {IMBH }}^{2}
$$

where $P_{\mathrm{ra}}$ is the probability that a cluster evolves to the runaway phase, $P_{\mathrm{IMBH}}$ is the probability that the runaway leads to IMBH formation and $P_{\text {merg }}$ is the probability that the cluster merges with another cluster. For Fregeau et al. (2006), it is simply $P_{\text {bin }}=P_{\text {ra }} P_{\mathrm{IMBH}}$. Eq. 5 is an oversimplification because the various factors are not independent of each other; 
for instance, $D_{\max }$ is larger for more massive IMBHs, which probably introduces a dependence on $M_{\mathrm{cl}}$. Also the cosmological evolution of the cluster formation rate should be taken into account. Fregeau et al. (2006) present a much more rigorous computation but we can use the above equations to derive our rate estimate from their results, $\Gamma \approx P_{\text {merg }} P_{\text {ra }} \Gamma_{\text {Fregeau }}$ with $\Gamma_{\text {Fregeau }} \approx 40-50\left(P_{\mathrm{ra}} / 0.1\right) \mathrm{yr}^{-1}$.

We take the same optimistic view as Fregeau et al. that VMSs always form IMBHs, $P_{\mathrm{IMBH}}=1$. The extra $P_{\mathrm{ra}}$ factor indicates that both clusters must contain an IMBH. The conditions for runaway are relatively well understood (Gürkan et al. 2004; Portegies Zwart et al. 2004; Freitag et al. 2006b; Gürkan et al. 2006) but the value of $P_{\text {ra }}$ is highly uncertain because these conditions apply to the initial cluster properties. Fregeau et al. choose $P_{\mathrm{ra}}=0.1$ as an illustrative value. We note that if all clusters are born with a concentration as high as a $W_{0}=8 \mathrm{King}$ model and the mass and half-mass radii of observed local globular clusters are similar to their initial values, $P_{\text {ra }}$ may be about 0.5 (Freitag et al. 2006c). On the other hand, Baumgardt et al. (2004) have argued that Galactic globular clusters containing an IMBH may be amongst the least dense clusters (for their mass) as a result of the strong IMBH-powered gravothermal expansion (see also Freitag et al. 2006a). The relatively small number of observed low-density clusters would therefore suggest a smaller $P_{\text {ra }}$ but the galactic tidal field may prevent the expansion of the cluster.

The factor $P_{\text {merg }}$ is also uncertain. As mentioned in the introduction, observational and theoretical points support the possibility of a large fraction of clusters being born in binaries, suggesting $P_{\text {merg }}=0.1-1$. Furthermore, if the scenario of Fellhauer \& Kroupa holds, each UCDG would require $\sim 10-100$ cluster-cluster mergers. UCDGs may be as numerous as normal galaxies, at least in galaxy clusters (e.g., Hilker \& Mieske 2004; Drinkwater et al. 2005). Hence the number of clusters that have been incorporated into UCDGs may be of the same order of magnitude as the number of those that have survived as isolated clusters (possibly after a binary merger). This indicates that the rate estimate for the "UCDG channel" should be similar (given the considerable uncertainties) to that of the "binary-merger channel". Finally, we find that the detection rate estimate for LISA is of

$$
\Gamma=4-5\left(P_{\mathrm{ra}} / 0.1\right)^{2} P_{\text {merg }} \mathrm{yr}^{-1} \text {. }
$$

Because of its superior sensibility at frequencies higher than $\sim 10^{-3} \mathrm{~Hz}$, the $B B O$ should be sensitive to IMBH binary coalescences from redshifts to at least $\sim 15$. A high merger rate could be envisaged as a potential confusion foreground for this mission. Fortunately, following similar arguments as discussed in Cutler \& Harms (2006) regarding the contribution of neutron star mergers, IMBH binaries should be easy to subtract out (C. Cutler personal communication). Thus the $B B O$ will open a new window of possibilities for our understanding of astrophysical scenarios leading to coalescence of IMBH binaries.

\section{ACKNOWLEDGMENTS}

We thank Leor Barack, Curt Cutler and Jonathan Gair for enlightening discussions and Cole Miller for helping us improving the manuscript. We acknowledge the Astronomisches Rechen-Institut for the computing resources on the GRACE cluster of the Volkswagen Foundation, SFB439. The work of PAS has been supported in the framework of the Third Level Agreement between the DFG and the IAC (Instituto de Astrofísica de Canarias). The work of MF is funded through the PPARC rolling grant at the Institute of Astronomy (IoA) in Cambridge. PAS is indebted with the IoA for inviting him the month of February 2006, where MF and he started working on this project.

\section{REFERENCES}

Aarseth S. J., 2003, Gravitational N-Body Simulations. Cambridge University Press

Barack L., Cutler C., 2004, Phys. Rev. D, 69, 082005

Baumgardt H., Makino J., Ebisuzaki T., 2004, ApJ, 613, 1143

Bekki K., Beasley M. A., Forbes D. A., Couch W. J., 2004, ApJ, 602, 730

Bender P. L., Hils D., 1997, Classical and Quantum Gravity, 14, 1439

Cutler C., Harms J., 2006, Phys. Rev. D, 73, 042001

Dieball A., Müller H., Grebel E. K., 2002, A\&A, 391, 547

Drinkwater M. J., Evstigneeva E., Gregg M. D., Jones J. B., Phillipps S., Jurek

R., 2005, in IAU Colloq. 198: Near-fields cosmology with dwarf elliptical galaxies, Jerjen H., Binggeli B., eds., pp. 398-403

Fellhauer M., Kroupa P., 2002, MNRAS, 330, 642

,- 2005, MNRAS, 359, 223

Finn L. S., Thorne K. S., 2000, Phys. Rev. D, 62, 124021

Fregeau J. M., Larson S. L., Miller M. C., O’Shaughnessy R., Rasio F. A., 2006, Observing IMBH-IMBH Binary Coalescences via Gravitational Radiation. preprint, astro-ph/0605732

Freitag, M., Amaro-Seoane, P., \& Kalogera, V. 2006a, Stellar Remnants in Galactic Nuclei: Mass Segregation., ApJ, 649, 91

Freitag M., Gürkan M. A., Rasio F. A., 2006b, MNRAS, 368, 141

Freitag, M., Rasio, F. A., \& Baumgardt, H. 2006c, MNRAS, 368, 121

Fujimoto M., Kumai Y., 1997, AJ, 113, 249

Fukushige T., Makino J., Kawai A., 2005, PASJ, 57, 1009

Gürkan M. A., Freitag M., Rasio F. A., 2004, ApJ, 604, 632

Gürkan M. A., Fregeau J. M., Rasio F. A., 2006, ApJ Lett., 640, L39

Gallagher S. C., Charlton J. C., Hunsberger S. D., Zaritsky D., Whitmore B. C. 2001, AJ, 122, 163

Han C., Ryden B. S., 1994, ApJ, 433, 80

Hilker M., Mieske S., 2004, in Baryons in Dark Matter Halos, Dettmar R., Klein U., Salucci P., eds.
Kontizas E., Kontizas M., Sedmak G., Smareglia R., 1989, AJ, 98, 590

Kontizas E., Kontizas M., Sedmak G., Smareglia R., Dapergolas A., 1990, AJ, 100,425

Larson S. L., 2003, Online sensitivity curve generator. http://www.srl.caltech.edu/ shane/sensitivity/

Larson S. L., Hiscock W. A., Hellings R. W., 2000, Physical Review D, 62, 062001

Merritt D., Milosavljević M., 2005, Living Reviews in Relativity, 8, 8

Mikkola, S. \& Aarseth, S. 2002, Celestial Mechanics and Dynamical Astronomy, 84, 343

Peters P. C., 1964, Physical Review, 136, 1224

Peters P. C., Mathews J., 1963, Physical Review, 131, 435

Phinney E. S., 2002, LISA Science Requirements. available on-line at www.its.caltech.edu/ esp/lisa/ LISTwg1.req-pr.pdf

Portegies Zwart S. F., Baumgardt H., Hut P., Makino J., McMillan S. L. W., 2004, Nat, 428, 724

Portegies Zwart S. F., McMillan S. L. W., 2000, ApJ Lett., 528, L17

Quinlan G. D., 1996, New Astronomy, 1, 255

Sesana A., Haardt F., Madau P., Volonteri M., 2004, ApJ, 611, 623

Theis C., 2002, Ap\&SS, 281, 97

van den Bergh S., 1991, ApJ, 369, 1

van der Marel, R. P. 2004, in Coevolution of Black Holes and Galaxies, from the Carnegie Observatories Centennial Symposia., ed. L. Ho (Cambridge University Press), 37

Whitmore B. C., Zhang Q., Leitherer C., Fall S. M., Schweizer F., Miller

B. W., 1999, AJ, 118, 1551

Zhang Q., Fall S. M., 1999, ApJ Lett., 527, L81 